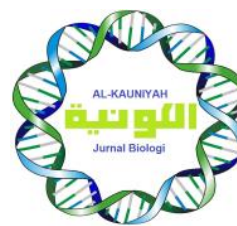

Available online at AL-KAUNIYAH: Jurnal Biologi

Website: http://journal.uinjkt.ac.id/index.php/kauniyah

AL-KAUNIYAH: Jurnal Biologi, 14(1), 2021, 162-183

PENGETAHUAN TUMBUHAN OBAT MASYARAKAT ADAT KAMPUNG DUKUH, GARUT, JAWA BARAT

\title{
MEDICINAL KNOWLEDGE OF TRADITIONAL COMMUNITY IN KAMPUNG DUKUH, GARUT REGENCY, WEST JAVA
}

\author{
Santhyami $^{1 *}$, Endah Sulistyawati ${ }^{2}$ \\ ${ }^{1}$ Department of Biology Education, Faculty of Teacher Training and Education, Universitas Muhammadiyah Surakarta. \\ Jl. A. Yani, Mendungan, Pabelan, Subdistrict of Kartasura, Sukoharjo Regency, Central Java, 57162 \\ ${ }^{2}$ School of Life Sciences and Technology, Institut Teknologi Bandung, Jalan Ganesha 10, Bandung 40132, West Java, \\ Indonesia \\ *Corresponding author: san915@ums.ac.id
}

Naskah Diterima: 15 Agustus 2020; Direvisi: 2020; Disetujui: 11 Maret 2021

\begin{abstract}
Abstrak
Masyarakat adat Kampung Dukuh, Cikelet, Kabupaten Garut, Jawa Barat masih mempertahankan tradisi leluhurnya terutama dalam pengobatan tradisional. Penelitian ini bertujuan untuk mendokumentasikan tumbuhan obat yang digunakan oleh masyarakat Kampung Dukuh. Metode yang digunakan adalah pendekatan etnobotani. Data yang dikumpulkan adalah daftar tumbuhan obat yang diketahui, lokasi tempat diperoleh atau ditanam, dan kegunaan masing-masing jenis tumbuhan tersebut. Identifikasi dilakukan di Herbarium Bandungense SITH-ITB. Lokalitas dan kegunaan masing-masing tanaman obat dikumpulkan dengan menggunakan wawancara semi terstruktur. Hasil penelitian menunjukkan bahwa masyarakat Kampung Dukuh mengklasifikasikan penyakit menjadi tiga yaitu penyakit biasa, penyakit karena sihir, dan penyakit yang disebabkan oleh makanan. Sebanyak 131 jenis tumbuhan dari 51 suku tercatat dimanfaatkan masyarakat sebagai obat. Lima suku dengan jumlah spesies terbanyak adalah Zingiberaceae, Poaceae, Asteraceae, Fabaceae, dan Solanaceae. Jenis penyakit yang disembuhkan dengan memanfaatkan tanaman obat yang paling banyak adalah perawatan sebelum dan sesudah melahirkan. Masyarakat memperoleh tanaman dari lima lokasi: kebon (kebun), leuweung (hutan), halaman rumah, pinggir jalan, dan huma (lahan pertanian kering). Hasil penelitian ini menunjukkan bahwa masyarakat Kampung Dukuh mengintegrasikan budaya penggunaan tumbuhan obat dengan upaya pelestarian keanekaragaman hayati setempat.
\end{abstract}

Kata kunci: Etnobotani; Kampung Dukuh; Pengetahuan tradisional; Tumbuhan obat

Abstract

Traditional community of Kampung Dukuh, in Cikelet, Garut Regency, West Java still keep their ancestral tradition alive, especially the traditional healing. This study aims to document the medicinal plants used by the people of Kampung Dukuh. Method used was ethnobotanical approach. Data collected were the list of medicinal plants known, locations the species obtained or planted, and the utility of each species. Identification was done at Herbarium Bandungense SITH-ITB. Locality and the utility of each medicinal plant were collected by using the semi-structured interview. The result showed that people of Kampung Dukuh classified illness into three: common illness, illness by magic and disease caused by food. A total of 131 species from 51 families of plants were recorded to be recognized and used by the community as medicines. Five families with the most number of species were Zingiberaceae, Poaceae, Asteraceae, Fabaceae, and Solanaceae. Type of medical condition mostly by utilizing medicinal plants was pre and postpartum care. People obtained plants from five locations; kebon (garden), leuweung (forest), buruan (home garden), sidewalk, and huma (dry farm). This research indicated that people of Kampung Dukuh integrated the culture of using medicinal plant with conservation effort of local biodiversity.

Keywords: Ethnobotany; Medicinal plants; Kampung Dukuh; Traditional knowledge

Permalink/DOI: http://dx.doi.org/10.15408/kauniyah.v14i1.16970 


\section{INTRODUCTION}

For a long time, humankind has always been using medicinal plants as conventional medicine to heal diverse ailments. Approximately 30,000 of a total 40,000 species of medicinal plants known in the world are allegedly located in Indonesia (Munadi, 2017). Medicinal plants have played an important role in managing a variety of healthcare and diseases in Indonesia. In some developing countries like Indonesia, people continue to use natural plants, especially in meeting basic health needs (Hernani, 2011).

Indonesia has the traditional healing culture known for hundreds of years ago which is passed down through generations. For instance, jamu, a traditional medicinal tonic, is widely known by Indonesian people. Footprints of traditional use of medicinal plants in Indonesia are well captured in the ancient original manuscript like Husodo Lontar Leaf (Java island) (Gunawan, Ramadhan, Iskandar, \& Partasamita, 2018), Usada (Bali island) (Suatama, 2019), Lontarak Pabbura (South Sulawesi) (Kusumah, 2017), Primbon Jampi Fibre document and Fibre of Blend Boreh Wulang nDalem. Some authentic proofs of traditional medicinal processing are shown on the relief of Borobudur wall, one of the most stellar temples in Indonesia (Sari, 2006).

Modernization is the ultimate obstacle in preserving the sustainability of traditional medicinal knowledge. Culture erosion can lead to the loss of traditional knowledge of traditional people (Bodeker, 2000; Brosi, Balick, Wolkou, Lee, \& Kostka, 2007; Ramirez, 2007). Other factors related to the expedite loss of traditional knowledge are the loss of biological resources, cultural homogenization and the disappearance of practices involving these plants (Balick, 2007), also increased accessibility to public health cares and improved transportation infrastructure (Weckmüller, Barriocanal, Maneja, \& Boada, 2019). The dissapearance of traditional medicinal knowledge, particularly on plants utilization is due to the fact that it is only passed through generations orally and has remained unwritten (Kaido, Veale, Havlik, \& Rama, 1997; Rahman, Fardusi, \& Reza, 2011). In fact, the depletion of intergenerational traditional knowledge has occurred in community living in Kampung Kuta, Ciamis, West Java Indonesia. Traditional knowledge of local people in Kampung Kuta in recognizing the medicinal plants was threatened to be extinct (Dwiartama, 2005). Hence, the documentation of traditional knowledge of medicinal plants is needed.

Sundanese ethnic in West Java, one of many ethnic groups in Indonesia, has been practiced traditional medicinal knowledge daily (Gunawan et al., 2018). One of the traditional communities of Sunda ethnic that still keeps their ancestral tradition alive is Kampung Dukuh community in Cikelet, Garut Regency, West Java Province. Kampung Dukuh is surrounded by natural resources, thus there is a strong relationship between people of Kampung Dukuh and nature. They keep many routine rituals to respect nature, such as Ngahaturan tuang (offering food to ancestor), Nyanggakeun (offering some farm yields to kuncen-sacred forest caretaker), Tilu waktos (offering food for the earth by kuncen) and many more. The community is dependent and respecting nature. Furthermore, the community obeys five ancestral principles related to nature, namely larangan (sacred forest), titipan (nature is entrusted by an ancestor), tutupan (keeping tree cover around the hamlet), garapan (land allowed to be cultivated), and cadangan (reserved land). The essence of these principles is that people may still use nature but still respect and maintain the carrying capacity of nature itself. This study aims to document and explore the knowledge of traditional people of Kampung Dukuh in recognizing and processing medicinal plants for the treatment of various ailments. The record of these plants and their uses will provide baseline data for future phytochemical and pharmacological studies.

\section{MATERIALS AND METHODS Study Area}

Figure 1 shows the map of Kampung Dukuh. It is situated on south part of Garut Regency, part of the south coast of Java Island. Kampung Dukuh is administratively located in Cijambe hamlet, Cikelet area, Garut Regency, West Java Indonesia. The distance between Kampung Dukuh and the capital of Garut Regency is about $100 \mathrm{~km}$ or $160 \mathrm{~km}$ from 
Bandung (capital of West Java Province) to the south. The altitude of Kampung Dukuh is 390 $\mathrm{m}$ above sea level. Kampung Dukuh is located on the sloping land of Dukuh mountain. The zone is between latitudes of $7-8^{\circ} \mathrm{S}$ and $70-108$ ${ }^{\circ} \mathrm{E}$. The average temperatures is $26{ }^{\circ} \mathrm{C}$. Kampung Dukuh covers 10 ha area, consist of Dukuh Landeuh (outer hamlet) (7 ha), Dukuh Tonggoh (inner hamlet) (1 ha), and bare land (2 ha). Most of the inhabitants are farmers.

\section{Ethnobotanical Data Collection}

The method of this study was descriptive qualitative with ethnobotanical approach. The initial interview was done to explore the daily basis of the community. Through this interview, we selected informants purposively (Martin, 1995). Based on this interview, we chose significant figures as prospective informants such as traditional leader, kuncen (forest caretaker), and paraji (midwife and health practitioner as well). We were also involved in activities done by some informants, such as working at garden and forest, attending rituals and observing the midwife on duty but we did not participate in all of the daily activities of the informant.

An open-ended interview with those three significant respondents was done during the initial data collection process. We started interactions with these respondents by first explaining the aims and objectives of the research to solicit their consent and cooperation before any ethnobotanical data were gathered. During these interviews, we emphasized the immense value which each significant figure's contribution could make to the compilation of a record of traditional knowledge of medicinal plants in this community. Data of initial prospective informant candidates were collected through the recommendation of these figures. Next candidates were obtained through snowball sampling methods. The criteria recognized to recruit further informants were: 1) people according to the previous informant had knowledge and experience in using medicinal plants, 2) patients of paraji who had used medicinal plants and practiced traditional treatments, and 3) farmers who planted plants included in the criteria of medicinal plants. Based on these criteria, the informants were grouped into two community groups: 1) traditional and important figures in the region such as traditional leader, forest caretaker, midwife, health practitioner and elders, 2) general figures of all ages.

With the help of an interpreter, all interviews and discussions were conducted in Sunda halus, the local language. Ethnobotanical data were collected through two stages. First stage was an open interview. We collected the data of diseases or illnesses commonly suffered by the community. Furthermore, concerning each type of disease or illness, we asked what plant or plant mixture they use to cure them, which part of the plant they use, and how they process it/them into medicines. The data on this stage were gathered from 16 people altogether which included customary figures as kuncen and respected elders, healer figures as shaman and midwife, and general residents of all ages. The second stage was the reverse of the first stage. At this stage, all informants were firstly asked to mention all plants used for medicinal purpose. On each type of plant, we then asked the use of plants related to medicinal purposes. The total informant in this stage was 10 people of the same array.

\section{Plant Collection}

Photograph and specimen of some unrecognized plants in the field were collected in situ for proper identification. The specimens were collected from both natural vegetation and garden such forest, home gardens and farm with the help of key informants. Data on each plant were recorded by using the data capture form.

\section{Plant Identification}

Some of the medicinal plants were identified in the field and the remaining ones were identified through literature review. Collected specimens were identified in Herbarium Bandungense, School of Life Science and Technology, Institut Teknologi Bandung. The identity and nomenclature of each plant species were determined following Backer and van den Brink (1965), Nielsen (1992), Prawira (1976), Ochse and van den Brink (1931), and Ogata et al. (1995). 


\section{Data Analysis}

The data of interview with informants were analyzed by medical anthropology approach while medicinal plant data were analyzed through medicinal ethnobotany approach (Martin, 1995). Both data were shown descriptively and quantitatively in tables and graphs. Medical anthropology aspect covered the cultural aspect related to the classification of diseases and illnesses based on the traditional community (disease concept), type of curing methods, the role of the traditional healer on the community, and how the knowledge was transferred through generations. Medicinal ethnobotany covered the identification of medicinal plants known and used by the people, their local names, part of the plant used, medicinal properties based on their experience, and the location where they were obtained.

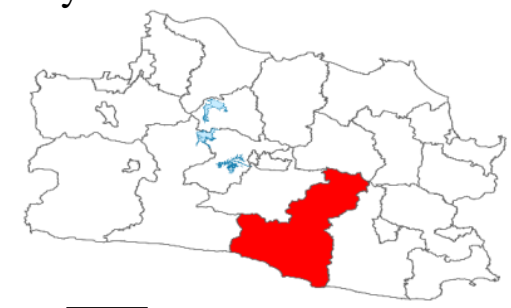

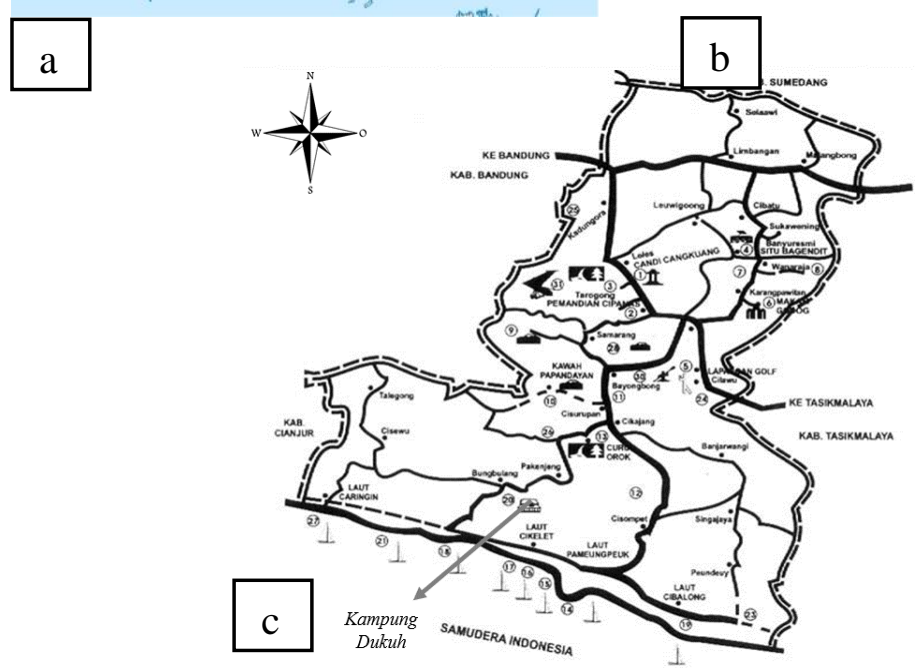

Figure 1. Research location: West Java Province (a), Garut Regency (b), and Location of Kampung Dukuh (c)

\section{RESULT}

\section{Medicinal plant}

The results showed that people of Kampung Dukuh recognized 131 species of medicinal plants from 51 families. Families with the most number of species are
Zingiberaceae (14 species), Poaceae (8 species), Asteraceae (7 species), Fabaceae (6 species), and Solanaceae (6 species). Table 1 shows various plant species used by people of Kampung Dukuh as medicine.

Table 1. List of medicinal plant species known by people of Kampung Dukuh

\begin{tabular}{|c|c|c|c|c|}
\hline Family & Scientific name & Vernacular name & $\begin{array}{c}\text { Ailment } \\
\text { treated }\end{array}$ & $\begin{array}{l}\text { Plant part used and } \\
\text { processing }\end{array}$ \\
\hline Amaranthaceae & Iresine herbstii Hook.F. & Beungbeureuman & Nausea & $\begin{array}{l}\text { Water decoction of } \\
\text { leaves }\end{array}$ \\
\hline \multirow[t]{3}{*}{ Amaryllidae } & Crinum asiaticum $\mathrm{L}$. & Bakung & Boil & $\begin{array}{l}\text { Root is shredded, } \\
\text { taped to boils }\end{array}$ \\
\hline & & & Heatiness & $\begin{array}{l}\text { Squeezed leaves } \\
\text { smeared on the back }\end{array}$ \\
\hline & Alium cepa $\mathrm{L}$. & Bawang beureum & $\begin{array}{l}\text { Common cold, } \\
\text { fever, flu }\end{array}$ & $\begin{array}{l}\text { Crushed bulbs are } \\
\text { rubbed on stomach } \\
\text { and head }\end{array}$ \\
\hline
\end{tabular}




\begin{tabular}{|c|c|c|c|c|}
\hline Family & Scientific name & Vernacular name & $\begin{array}{c}\text { Ailment } \\
\text { treated }\end{array}$ & $\begin{array}{l}\text { Plant part used and } \\
\text { processing }\end{array}$ \\
\hline & & & $\begin{array}{l}\text { Digestive } \\
\text { health }\end{array}$ & $\begin{array}{l}\text { Crushed bulbs are } \\
\text { mixed with sugar }\end{array}$ \\
\hline & & & Eczema & $\begin{array}{l}\text { Crushed bulbs are } \\
\text { rubbed into the skin }\end{array}$ \\
\hline & Allium sativum $\mathrm{L}$. & Bawang bodas & Hypertension & $\begin{array}{l}\text { Crushed bulb is eaten } \\
\text { directly }\end{array}$ \\
\hline & & & $\begin{array}{l}\text { Digestive } \\
\text { health }\end{array}$ & $\begin{array}{l}\text { Crushed bulb is mixec } \\
\text { with sugar }\end{array}$ \\
\hline & & & Toothache & $\begin{array}{l}\text { Bulbs are chewed, } \\
\text { placed on cavities }\end{array}$ \\
\hline Anacardiaceae & Mangifera indica $\mathrm{L}$. & Mangga & Hypertension & Fruit is eaten directly \\
\hline \multirow[t]{3}{*}{ Annonaceae } & Annona muricata $\mathrm{L}$. & Nangka walanda & $\begin{array}{l}\text { Running cold } \\
\text { in children }\end{array}$ & $\begin{array}{l}\text { Pounded leaves are } \\
\text { smeared on the head }\end{array}$ \\
\hline & & & $\begin{array}{l}\text { Fever, body } \\
\text { ache }\end{array}$ & $\begin{array}{l}\text { Water decoction of } \\
\text { leaves }\end{array}$ \\
\hline & & & Vertigo & $\begin{array}{l}\text { Water decoction is } \\
\text { applied to the body }\end{array}$ \\
\hline \multirow[t]{4}{*}{ Apiaceae } & $\begin{array}{l}\text { Centella asiatica }(\mathrm{L} .) \\
\text { Urb. }\end{array}$ & Antanan & $\begin{array}{l}\text { Hypertension, } \\
\text { body ache }\end{array}$ & $\begin{array}{l}\text { Water decoction of } \\
\text { leaves }\end{array}$ \\
\hline & & & $\begin{array}{l}\text { Problem in } \\
\text { respiratory } \\
\text { system }\end{array}$ & $\begin{array}{l}\text { Dried leaves are } \\
\text { served as herbal drink }\end{array}$ \\
\hline & Apium graveolens L. & Seledri & Hypertension & $\begin{array}{l}\text { Water decoction of } \\
\text { leaves }\end{array}$ \\
\hline & Daucus carota L. & Wortel & Sore eyes & Root is eaten directly \\
\hline \multirow[t]{4}{*}{ Apocynaceae } & $\begin{array}{l}\text { Allamanda cathartica } \\
\text { L. }\end{array}$ & Lame areuy & $\begin{array}{l}\text { Tonic, } \\
\text { diarrhea, ulcer }\end{array}$ & $\begin{array}{l}\text { Water decoction of } \\
\text { stem }\end{array}$ \\
\hline & & & $\begin{array}{l}\text { Post partum } \\
\text { care }\end{array}$ & $\begin{array}{l}\text { Powder of dried stem } \\
\text { is served as herbal } \\
\text { drink }\end{array}$ \\
\hline & $\begin{array}{l}\text { Alstonia scholaris (L.) } \\
\text { R.Br. }\end{array}$ & Lame hideung & $\begin{array}{l}\text { Ulcer, body } \\
\text { sore }\end{array}$ & $\begin{array}{l}\text { Water decoction of } \\
\text { stem }\end{array}$ \\
\hline & & & $\begin{array}{l}\text { Post partum } \\
\text { care }\end{array}$ & $\begin{array}{l}\text { Powder of dried stem } \\
\text { is served as herbal } \\
\text { drink }\end{array}$ \\
\hline \multirow[t]{3}{*}{ Araceae } & $\begin{array}{l}\text { Colocasia esculenta } \\
\text { (L.) Schott. }\end{array}$ & Talas bolang & Cough & $\begin{array}{l}\text { The sap from cut stem } \\
\text { is collected, left } \\
\text { overnight }\end{array}$ \\
\hline & $\begin{array}{l}\text { Xanthosoma ningrum } \\
\text { (Vell.) Masf. }\end{array}$ & Taleus hideung & Bruised skin & $\begin{array}{l}\text { Squeezed leaves are } \\
\text { smeared on bruise }\end{array}$ \\
\hline & $\begin{array}{l}\text { Colocasia gigantea } \\
\text { (BI.) Hook.f. }\end{array}$ & Taleus kajar-kajar & Cough & $\begin{array}{l}\text { The sap from cut stem } \\
\text { is collected, left } \\
\text { overnight }\end{array}$ \\
\hline \multirow[t]{3}{*}{ Arecaceae } & Areca catechu $\mathrm{L}$. & Jambe & $\begin{array}{l}\text { Dental health, } \\
\text { throat sore }\end{array}$ & Root is chewed up \\
\hline & & & Ulcer & $\begin{array}{l}\text { Hot water is poured } \\
\text { into sliced nut }\end{array}$ \\
\hline & & & $\begin{array}{l}\text { Erectile } \\
\text { dysfunction, } \\
\text { tonic }\end{array}$ & $\begin{array}{l}\text { Nut is eaten, water } \\
\text { decoction of root }\end{array}$ \\
\hline
\end{tabular}




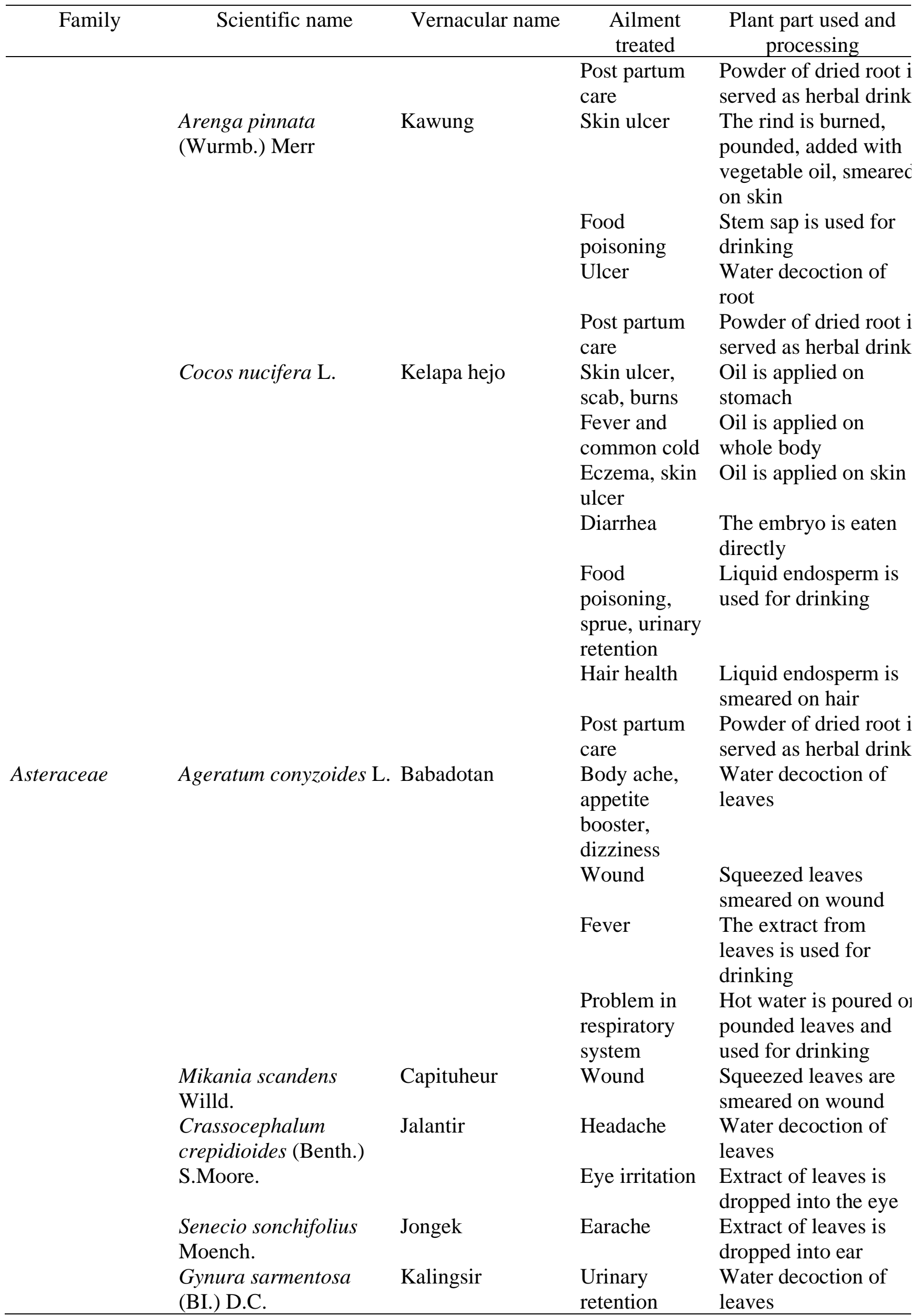




\begin{tabular}{|c|c|c|c|c|}
\hline Family & Scientific name & Vernacular name & $\begin{array}{c}\text { Ailment } \\
\text { treated }\end{array}$ & $\begin{array}{l}\text { Plant part used and } \\
\text { processing }\end{array}$ \\
\hline & Eupatorium odoratum & $\mathrm{Ki}$ andih & Nose bleeding & $\begin{array}{l}\text { Squeezed leaves are } \\
\text { put into nostril }\end{array}$ \\
\hline & & & Wound & $\begin{array}{l}\text { Squeezed leaves are } \\
\text { smeared on wound }\end{array}$ \\
\hline & & & Tonic & $\begin{array}{l}\text { Water decoction of } \\
\text { leaves }\end{array}$ \\
\hline & $\begin{array}{l}\text { Blumea balsamifera } \\
\text { (L.) D.C. }\end{array}$ & Sembung & $\begin{array}{l}\text { Appetite } \\
\text { booster }\end{array}$ & $\begin{array}{l}\text { Dried leaves are boile } \\
\text { and served as tea }\end{array}$ \\
\hline & & & $\begin{array}{l}\text { Body ache, } \\
\text { post partum } \\
\text { care, tonic }\end{array}$ & $\begin{array}{l}\text { Water decoction of } \\
\text { leaves }\end{array}$ \\
\hline \multirow[t]{3}{*}{ Bignoniaceae } & $\begin{array}{l}\text { Dolichandrone } \\
\text { spathecea (L.f.) K. } \\
\text { Schum. }\end{array}$ & Kalujaran & Cough & $\begin{array}{l}\text { The sap from cut stem } \\
\text { is collected and used } \\
\text { for drinking }\end{array}$ \\
\hline & & & Sore eyes & $\begin{array}{l}\text { Extract of leaves is } \\
\text { dropped into the eye }\end{array}$ \\
\hline & & & $\begin{array}{l}\text { Post partum } \\
\text { care }\end{array}$ & Boiled leaves are eate \\
\hline Bombacaceae & $\begin{array}{l}\text { Ceiba petandra (L.) } \\
\text { Gaertn. }\end{array}$ & Randu & $\begin{array}{l}\text { Pre partum } \\
\text { care }\end{array}$ & $\begin{array}{l}\text { The extract from } \\
\text { leaves is used for } \\
\text { drinking }\end{array}$ \\
\hline Brassicaceae & $\begin{array}{l}\text { Rorippa indica } \\
\text { (L.)Riern. }\end{array}$ & Sawi hayam & Body ache & $\begin{array}{l}\text { Water decoction of } \\
\text { leaves }\end{array}$ \\
\hline Burmanniaceae & $\begin{array}{l}\text { Vanilla planifolia } \\
\text { Andrews. }\end{array}$ & Anggrek panili & Toothache & $\begin{array}{l}\text { Flower is chewed, } \\
\text { placed on cavities }\end{array}$ \\
\hline \multirow[t]{4}{*}{ Caesalpiniaceae } & Tamarindus indica L. & Asam & Headache & $\begin{array}{l}\text { Water decoction of } \\
\text { fruit }\end{array}$ \\
\hline & & & Skincare & $\begin{array}{l}\text { Powder of dried rind } \mathrm{i} \\
\text { used as mask }\end{array}$ \\
\hline & & & Dizziness & $\begin{array}{l}\text { Hot water is poured ol } \\
\text { fruit, added with suga } \\
\text { and used for drinking }\end{array}$ \\
\hline & Senna alata (L.) Roxb. & Ki manila & $\begin{array}{l}\text { Skin ulcer, } \\
\text { scab }\end{array}$ & $\begin{array}{l}\text { Pounded leaves are } \\
\text { smeared on skin }\end{array}$ \\
\hline \multirow[t]{7}{*}{ Caricaceae } & Carica papaya L. & Gedang & $\begin{array}{l}\text { Hypotension, } \\
\text { breast milk } \\
\text { booster }\end{array}$ & Boiled leaves are eate \\
\hline & & & Insect bites & $\begin{array}{l}\text { The sap of stem and } \\
\text { leaves are dropped to } \\
\text { the wound }\end{array}$ \\
\hline & & & $\begin{array}{l}\text { Body ache, } \\
\text { tonic }\end{array}$ & $\begin{array}{l}\text { Water decoction of } \\
\text { leaves }\end{array}$ \\
\hline & & & Toothache & $\begin{array}{l}\text { Leaves sap is dropped } \\
\text { into cavity }\end{array}$ \\
\hline & & & Headache & $\begin{array}{l}\text { Ponded seed is } \\
\text { smeared on head }\end{array}$ \\
\hline & & & $\begin{array}{l}\text { Post partum } \\
\text { care }\end{array}$ & $\begin{array}{l}\text { Powder of dried root } \mathrm{i} \\
\text { served as herbal drink }\end{array}$ \\
\hline & & & $\begin{array}{l}\text { Constipation, } \\
\text { sprue }\end{array}$ & Fruit is eaten directly \\
\hline
\end{tabular}




\begin{tabular}{|c|c|c|c|c|}
\hline Family & Scientific name & Vernacular name & $\begin{array}{c}\text { Ailment } \\
\text { treated }\end{array}$ & $\begin{array}{l}\text { Plant part used and } \\
\text { processing }\end{array}$ \\
\hline Clusiaceae & $\begin{array}{l}\text { Garcinia Mangostana } \\
\text { L. }\end{array}$ & Manggu & Skin ulcer & $\begin{array}{l}\text { Fruit is burned, } \\
\text { crushed, added with } \\
\text { coconut oil, smeared } \\
\text { on skin }\end{array}$ \\
\hline Convolvulaceae & $\begin{array}{l}\text { Ipomoea aquatica } \\
\text { Forsk. }\end{array}$ & Kangkung & $\begin{array}{l}\text { Hypotension, } \\
\text { insomnia }\end{array}$ & Boiled leaves are eate \\
\hline \multirow[t]{5}{*}{ Cucurbithaceae } & Cucumis sativus L. & Bonteng & $\begin{array}{l}\text { Heatiness, } \\
\text { hypertension, } \\
\text { headache }\end{array}$ & Fruit is eaten directly \\
\hline & $\begin{array}{l}\text { Lagenaria leucantha } \\
\text { (Duch.) Poir }\end{array}$ & Kukuk & $\begin{array}{l}\text { Erectile } \\
\text { dysfunction } \\
\text { Ulcer }\end{array}$ & $\begin{array}{l}\text { Pounded leaves are } \\
\text { smeared on penis } \\
\text { Fruit is eaten directly }\end{array}$ \\
\hline & $\begin{array}{l}\text { Momordica charantia } \\
\text { L. }\end{array}$ & Paria & Itchy skin & $\begin{array}{l}\text { Pounded leaves are } \\
\text { smeared on skin }\end{array}$ \\
\hline & $\begin{array}{l}\text { Cucurbita moschata } \\
\text { (Duch.) Poir. }\end{array}$ & Waluh ageung & Ulcer & Boiled fruit is eaten \\
\hline & Sechium edule SW. & Waluh siam & Hypotension & Fruit is eaten directly \\
\hline \multirow[t]{9}{*}{ Euphorbiaceae } & Jatropha curcas L. & Jarak & Toothache & $\begin{array}{l}\text { Leaves sap is dropped } \\
\text { into cavity }\end{array}$ \\
\hline & Ricinus communis L. & Kaliki & $\begin{array}{l}\text { Post partum } \\
\text { care }\end{array}$ & $\begin{array}{l}\text { Leaves as warm } \\
\text { charcoal wrapper to si } \\
\text { on as vagina therapy }\end{array}$ \\
\hline & & & Earache & $\begin{array}{l}\text { Heated up stem is put } \\
\text { in the ear canal while } \\
\text { blown }\end{array}$ \\
\hline & $\begin{array}{l}\text { Sauropus androgynus } \\
\text { (L.) Merr. }\end{array}$ & Katuk & $\begin{array}{l}\text { Hypotension, } \\
\text { anemia, breast } \\
\text { milk booster, } \\
\text { sore eyes }\end{array}$ & Boiled leaves are eate \\
\hline & Euphorbia hirta L. & Nanangkaan & $\begin{array}{l}\text { Post partum } \\
\text { care - over } \\
\text { bleeding }\end{array}$ & $\begin{array}{l}\text { Water decoction of } \\
\text { leaves }\end{array}$ \\
\hline & $\begin{array}{l}\text { Manihot esculanta } \\
\text { Crantz. }\end{array}$ & Sampeu' & $\begin{array}{l}\text { Hypotension, } \\
\text { anemia }\end{array}$ & Boiled leaves are eate \\
\hline & & & Wound & $\begin{array}{l}\text { Squeezed leaves are } \\
\text { put into wound }\end{array}$ \\
\hline & & & Ulcer & $\begin{array}{l}\text { The tuber is eaten } \\
\text { directly }\end{array}$ \\
\hline & & & Heatiness & $\begin{array}{l}\text { Starch is boiled, mixe } \\
\text { with water and used } \\
\text { for drinking }\end{array}$ \\
\hline \multirow[t]{3}{*}{ Fabaceae } & $\begin{array}{l}\text { Gliricidia sepium } \\
\text { (Jacq.) Kunth ex Walp }\end{array}$ & Angrum & Skin disease & $\begin{array}{l}\text { Pounded leaves are } \\
\text { smeared on the skin }\end{array}$ \\
\hline & $\begin{array}{l}\text { Erythrina subumbrans } \\
\text { (Hassk.) Merr. }\end{array}$ & Dadap & Cough & $\begin{array}{l}\text { Grinded young leaves } \\
\text { are applied to the necl } \\
\text { as compress, the } \\
\text { extract of leaves is } \\
\text { used for drinking }\end{array}$ \\
\hline & & & $\begin{array}{l}\text { Intestinal } \\
\text { worm }\end{array}$ & $\begin{array}{l}\text { The extract of leaves } \mathrm{i} \\
\text { used for drinking }\end{array}$ \\
\hline
\end{tabular}




\begin{tabular}{|c|c|c|c|c|}
\hline Family & Scientific name & Vernacular name & $\begin{array}{c}\text { Ailment } \\
\text { treated }\end{array}$ & $\begin{array}{l}\text { Plant part used and } \\
\text { processing }\end{array}$ \\
\hline & & & $\begin{array}{l}\text { Fever in } \\
\text { children, body } \\
\text { ache }\end{array}$ & $\begin{array}{l}\text { Squeezed leaves are } \\
\text { smeared on body }\end{array}$ \\
\hline & & & $\begin{array}{l}\text { Near- } \\
\text { sightedness }\end{array}$ & $\begin{array}{l}\text { Stem sap is dropped } \\
\text { into the eyes }\end{array}$ \\
\hline & & & $\begin{array}{l}\text { Heatiness, } \\
\text { headache, } \\
\text { back pain, } \\
\text { hoarseness, } \\
\text { constipation }\end{array}$ & $\begin{array}{l}\text { Water decoction of } \\
\text { leaves }\end{array}$ \\
\hline & & & Sprue & $\begin{array}{l}\text { Squeezed leaves are } \\
\text { smeared on sprue }\end{array}$ \\
\hline & & & $\begin{array}{l}\text { Post partum } \\
\text { care }\end{array}$ & $\begin{array}{l}\text { Squeezed leaves are } \\
\text { smeared on stomach }\end{array}$ \\
\hline & $\begin{array}{l}\text { Vigna radiata }(\mathrm{L} .) \\
\text { Wilczek. }\end{array}$ & Kacang hejo & Fever & $\begin{array}{l}\text { Seeds are cooked as } \\
\text { porridge }\end{array}$ \\
\hline & $\begin{array}{l}\text { Desmodium triquetrum } \\
\text { D.C. }\end{array}$ & Ki congcorang & $\begin{array}{l}\text { Tonic, back } \\
\text { pain, ulcer }\end{array}$ & $\begin{array}{l}\text { Water decoction of } \\
\text { leaves }\end{array}$ \\
\hline & Abrus precatorius L. & Saga & Eye irritation & $\begin{array}{l}\text { Extract of leaves is } \\
\text { dropped into the eye }\end{array}$ \\
\hline & & & Boil & $\begin{array}{l}\text { Pounded leaves are } \\
\text { taped to boils }\end{array}$ \\
\hline & Arachis hypogaea L. & Suuk & Constipation & Boiled nut is eaten \\
\hline Iridaceae & $\begin{array}{l}\text { Belamcanda chinensis } \\
\text { (L.) D.C. }\end{array}$ & Suliga & Rib pain & $\begin{array}{l}\text { Pounded fruit is } \\
\text { smeared on rib }\end{array}$ \\
\hline \multirow[t]{5}{*}{ Lamiaceae } & $\begin{array}{l}\text { Coleus scutellaroides } \\
\text { (L.) Bth. }\end{array}$ & Jawer kotok & Sore eyes & $\begin{array}{l}\text { Squeezed leaves are } \\
\text { used as eye mask }\end{array}$ \\
\hline & Ocimum basilicum L. & Kemangi & Headache & $\begin{array}{l}\text { Water decoction of } \\
\text { leaves }\end{array}$ \\
\hline & $\begin{array}{l}\text { Orthosiphon aristatus } \\
\text { (BI.) Miq }\end{array}$ & Kumis kucing & $\begin{array}{l}\text { Diarrhea, } \\
\text { body ache, } \\
\text { back pain, } \\
\text { ulcer, tonic }\end{array}$ & $\begin{array}{l}\text { Water decoction of } \\
\text { leaves }\end{array}$ \\
\hline & & & $\begin{array}{l}\text { Urinary } \\
\text { retention }\end{array}$ & $\begin{array}{l}\text { Water decoction of } \\
\text { leaves and flower }\end{array}$ \\
\hline & Persea americana Mill. & Apuket & Body ache & $\begin{array}{l}\text { Water decoction of } \\
\text { leaves }\end{array}$ \\
\hline Loranthaceae & Scurrula sp. & $\begin{array}{l}\text { Mangandeuh } \\
\text { jengkol }\end{array}$ & Cancer & $\begin{array}{l}\text { Dried leaves are } \\
\text { served as herbal drink }\end{array}$ \\
\hline \multirow[t]{3}{*}{ Malvaceae } & Gossypium sp. & Kapas & Cold urticaria & $\begin{array}{l}\text { The raw fiber is } \\
\text { warmed up and rubber } \\
\text { on skin }\end{array}$ \\
\hline & Urena lobata L. & Pungpurutan & Wound & $\begin{array}{l}\text { Squeezed leaves are } \\
\text { smeared into wound }\end{array}$ \\
\hline & Sida rhombifolia $\mathrm{L}$. & Sadagori & Rheumatism & $\begin{array}{l}\text { Water decoction of } \\
\text { root }\end{array}$ \\
\hline Marantaceae & $\begin{array}{l}\text { Maranta arundinaceae } \\
\text { L. }\end{array}$ & Sagu & $\begin{array}{l}\text { Meal for } \\
\text { recovery period }\end{array}$ & Boiled root is eaten \\
\hline Melastomataceae & $\begin{array}{l}\text { Clidemia hirta (L.) } \\
\text { D.Don. }\end{array}$ & Harendong & $\begin{array}{l}\text { Post partum } \\
\text { care }\end{array}$ & $\begin{array}{l}\text { Powder of dried leave } \\
\text { is served as herbal }\end{array}$ \\
\hline
\end{tabular}




\begin{tabular}{|c|c|c|c|c|}
\hline Family & Scientific name & Vernacular name & $\begin{array}{l}\text { Ailment } \\
\text { treated }\end{array}$ & $\begin{array}{l}\text { Plant part used and } \\
\text { processing }\end{array}$ \\
\hline & & & Wound & $\begin{array}{l}\text { drink } \\
\text { Squeezed leaves are } \\
\text { smeared on wound }\end{array}$ \\
\hline \multirow[t]{2}{*}{ Meliaceae } & $\begin{array}{l}\text { Dysoxylum decandrum } \\
\text { Merr. }\end{array}$ & Ki tahi & $\begin{array}{l}\text { Diabetic } \\
\text { wound }\end{array}$ & $\begin{array}{l}\text { Pounded leaves are } \\
\text { smeared on wound }\end{array}$ \\
\hline & $\begin{array}{l}\text { Swietenia mahagoni } \\
\text { (L.) Jacq. }\end{array}$ & Mahoni & $\begin{array}{l}\text { Diabetes, } \\
\text { malaria, body } \\
\text { ache }\end{array}$ & Seed is eaten raw \\
\hline \multirow[t]{2}{*}{ Menispermaceae } & $\begin{array}{l}\text { Cyclea barbata (Wall.) } \\
\text { Miers. }\end{array}$ & Cingcau & Cold & $\begin{array}{l}\text { The sap of squeezed } \\
\text { leaves is clotted }\end{array}$ \\
\hline & $\begin{array}{l}\text { Tinospora tubreculata } \\
\text { Beumee. }\end{array}$ & Patrawalik & $\begin{array}{l}\text { Body ache, } \\
\text { late periods, } \\
\text { tonic }\end{array}$ & $\begin{array}{l}\text { Water decoction of } \\
\text { stem }\end{array}$ \\
\hline \multirow[t]{3}{*}{ Mimosaceae } & Parkia speciosa Hassk. & Peuteuy ageung & $\begin{array}{l}\text { Intestinal } \\
\text { worm }\end{array}$ & Fruit is eaten directly \\
\hline & & & Skin ulcer & $\begin{array}{l}\text { Leaves is chewed up, } \\
\text { applied on skin }\end{array}$ \\
\hline & $\begin{array}{l}\text { Leucaena glauca } \\
\text { Benth. }\end{array}$ & Peuteuy cina & $\begin{array}{l}\text { Intestinal } \\
\text { worm }\end{array}$ & Fruit is eaten directly \\
\hline \multirow[t]{3}{*}{ Moraceae } & $\begin{array}{l}\text { Artocarpus } \\
\text { heterophyllus Lmk. }\end{array}$ & Nangka & Body ache & $\begin{array}{l}\text { Water decoction of } \\
\text { leaves }\end{array}$ \\
\hline & $\begin{array}{l}\text { Artocarpus altilis } \\
\text { (Park.) Fosrberg. }\end{array}$ & Sukun & Cholesterol & $\begin{array}{l}\text { Leaves are dried, } \\
\text { brewed and used for } \\
\text { drinking }\end{array}$ \\
\hline & $\begin{array}{l}\text { Artocarpus elastica } \\
\text { Reinw. }\end{array}$ & Teureup & $\begin{array}{l}\text { Post partum } \\
\text { care }\end{array}$ & $\begin{array}{l}\text { The bark is wrapped } \\
\text { around the stomach }\end{array}$ \\
\hline Moringaceae & Moringa oleifera Lamk. & Kelor & Tonic & Boiled leaves are eate \\
\hline \multirow[t]{5}{*}{ Musaceae } & $\begin{array}{l}\text { Musa } \\
\text { acuminata Colla var.A } \\
\text { AA Group }\end{array}$ & Cau beureum & $\begin{array}{l}\text { Pre partum } \\
\text { care }\end{array}$ & $\begin{array}{l}\text { Leaves as warm } \\
\text { charcoal wrapper to } \\
\text { put on belly }\end{array}$ \\
\hline & & & Wound & $\begin{array}{l}\text { The sap from all part } \\
\text { of plant can be applier } \\
\text { on wound }\end{array}$ \\
\hline & $\begin{array}{l}\text { Musa acuminata Colla } \\
\text { var. Cavendish }\end{array}$ & Cau ambon & $\begin{array}{l}\text { Female } \\
\text { contraception, } \\
\text { ulcer } \\
\text { Cough }\end{array}$ & $\begin{array}{l}\text { Flower is chopped to } \\
\text { collect the sap and } \\
\text { used for drinking } \\
\text { Fruit is burned on } \\
\text { stove \& eaten }\end{array}$ \\
\hline & & & Wound & $\begin{array}{l}\text { Sap of stem is smeare } \\
\text { on wound }\end{array}$ \\
\hline & Musa eumusa var. AAB & Cau kapas & Earache & $\begin{array}{l}\text { The fruit is burned up } \\
\text { the steam is blown to } \\
\text { ear }\end{array}$ \\
\hline \multirow[t]{3}{*}{ Myrtaceae } & $\begin{array}{l}\text { Eugenia aromatica } \\
\text { O.K. }\end{array}$ & Cengkeh & Toothache & $\begin{array}{l}\text { The seed is chewed } \\
\text { and placed on cavity }\end{array}$ \\
\hline & & & $\begin{array}{l}\text { Post partum } \\
\text { care }\end{array}$ & $\begin{array}{l}\text { Powder of dried fruit } \\
\text { is served as herbal } \\
\text { drink }\end{array}$ \\
\hline & & & Nose bleeding & $\begin{array}{l}\text { Squeezed leaves are } \\
\text { put into nostril }\end{array}$ \\
\hline
\end{tabular}




\begin{tabular}{|c|c|c|c|c|}
\hline Family & Scientific name & Vernacular name & $\begin{array}{c}\text { Ailment } \\
\text { treated }\end{array}$ & $\begin{array}{l}\text { Plant part used and } \\
\text { processing }\end{array}$ \\
\hline & Psidium guajava $\mathrm{L}$. & Jambu batu & $\begin{array}{l}\text { Diarrhea, } \\
\text { headache, } \\
\text { stomachache }\end{array}$ & $\begin{array}{l}\text { Water decoction of } \\
\text { leaves }\end{array}$ \\
\hline & $\begin{array}{l}\text { Syzigium aqueum } \\
\text { (Burm.f.) Alst. }\end{array}$ & Jambu cai & Fever & $\begin{array}{l}\text { Water decoction of } \\
\text { leaves }\end{array}$ \\
\hline & $\begin{array}{l}\text { Eugenia polyantha } \\
\text { Wight. }\end{array}$ & Salam & Hypertension & $\begin{array}{l}\text { Water decoction of } \\
\text { leaves }\end{array}$ \\
\hline Oleaceae & $\begin{array}{l}\text { Jasminum sambac (L.) } \\
\text { Ait. }\end{array}$ & Melati & $\begin{array}{l}\text { Circumcision } \\
\text { care }\end{array}$ & Part of bathing ritual \\
\hline Oxalidaceae & Averrhoa carambola $\mathrm{L}$. & Balingbing & Hypertension & Fruit is eaten directly \\
\hline \multirow[t]{4}{*}{ Pedaliaceae } & $\begin{array}{l}\text { Andrographis } \\
\text { paniculata } \text { Ness }\end{array}$ & Sambilata & $\begin{array}{l}\text { Appetite } \\
\text { booster }\end{array}$ & $\begin{array}{l}\text { Water decoction of } \\
\text { leaves }\end{array}$ \\
\hline & $\begin{array}{l}\text { Malvaviscus arboreus } \\
\text { Cav. }\end{array}$ & Wera & Typhoid fever & $\begin{array}{l}\text { Pounded leaves are } \\
\text { smeared on body }\end{array}$ \\
\hline & Sesamum orientale L. & Wijen & Wound & $\begin{array}{l}\text { Oil from seed is } \\
\text { smeared on wound }\end{array}$ \\
\hline & & & Luxation & $\begin{array}{l}\text { Oil is used to massage } \\
\text { the affected part }\end{array}$ \\
\hline \multirow[t]{9}{*}{ Piperaceae } & $\begin{array}{l}\text { Piper sarmentosum } \\
\text { Roxb. Ex Hunter }\end{array}$ & Karuk & $\begin{array}{l}\text { Asthma, } \\
\text { asphyxiate } \\
\text { Cough }\end{array}$ & $\begin{array}{l}\text { Pounded leaves are } \\
\text { smeared on chest } \\
\text { Squeezed leaves are } \\
\text { smeared on neck }\end{array}$ \\
\hline & & & $\begin{array}{l}\text { Fever in } \\
\text { children }\end{array}$ & $\begin{array}{l}\text { Squeezed leaves are } \\
\text { smeared on whole } \\
\text { body }\end{array}$ \\
\hline & Piper aduncum L. & Ki seureuh & $\begin{array}{l}\text { Post partum } \\
\text { care }\end{array}$ & $\begin{array}{l}\text { Water decoction of } \\
\text { leaves }\end{array}$ \\
\hline & & & Nose bleeding & $\begin{array}{l}\text { Squeezed leaves are } \\
\text { put into nostril }\end{array}$ \\
\hline & Piper ningrum $\mathrm{L}$. & Pedes & Toothache & $\begin{array}{l}\text { Crushed seeds are put } \\
\text { into cavity }\end{array}$ \\
\hline & Piper betle L. & Seureuh & Tonsillitis & $\begin{array}{l}\text { Water decoction of } \\
\text { leaves }\end{array}$ \\
\hline & & & Nose bleeding & $\begin{array}{l}\text { Squeezed leaves are } \\
\text { put into nostril }\end{array}$ \\
\hline & & & $\begin{array}{l}\text { Dental care } \\
\text { Urinary } \\
\text { retention, birth } \\
\text { bleeding }\end{array}$ & $\begin{array}{l}\text { Leaves are chewed } \\
\text { Water decoction of } \\
\text { leaves }\end{array}$ \\
\hline & & & Sore eyes & $\begin{array}{l}\text { The extract of leaves } i \\
\text { dropped into the eyes }\end{array}$ \\
\hline \multirow[t]{3}{*}{ Poaceae } & $\begin{array}{l}\text { Dendrocalamus asper } \\
\text { (Schult.f.) Backer ex } \\
\text { Heyne }\end{array}$ & Awi bitung & Cough & $\begin{array}{l}\text { The sap from cut } \\
\text { bamboo shoot is } \\
\text { collected, served as } \\
\text { wine }\end{array}$ \\
\hline & & & Toothache & $\begin{array}{l}\text { Pounded bamboo } \\
\text { shoot is steamed, put } \\
\text { into the cavity }\end{array}$ \\
\hline & $\begin{array}{l}\text { Gigantochloa apus } \\
\text { Kurz }\end{array}$ & Awi tali & Cough & $\begin{array}{l}\text { The sap from cut } \\
\text { bamboo shoot is }\end{array}$ \\
\hline
\end{tabular}




\begin{tabular}{|c|c|c|c|c|}
\hline \multirow[t]{18}{*}{ Family } & Scientific name & Vernacular name & $\begin{array}{c}\text { Ailment } \\
\text { treated }\end{array}$ & $\begin{array}{l}\text { Plant part used and } \\
\text { processing }\end{array}$ \\
\hline & & & & $\begin{array}{l}\text { collected, served as } \\
\text { wine }\end{array}$ \\
\hline & $\begin{array}{l}\text { Schizostachyum blumei } \\
\text { Ness. }\end{array}$ & Awi tamiang & $\begin{array}{l}\text { Sharpen the } \\
\text { vision }\end{array}$ & $\begin{array}{l}\text { The sap of bamboo } \\
\text { shoot is dropped into } \\
\text { eyes }\end{array}$ \\
\hline & \multirow[t]{2}{*}{ Eleusine indica Gaertn } & \multirow[t]{2}{*}{ Carulang } & $\begin{array}{l}\text { Post partum } \\
\text { care }\end{array}$ & $\begin{array}{l}\text { Steamed leaves are } \\
\text { smeared over the } \\
\text { stomach }\end{array}$ \\
\hline & & & Hair loss & $\begin{array}{l}\text { Pounded leaves are } \\
\text { condensed and put on } \\
\text { the scalp }\end{array}$ \\
\hline & \multirow[t]{2}{*}{$\begin{array}{l}\text { Imperata cylindrica } \\
\text { (Ness) C.E.Hubb. }\end{array}$} & \multirow[t]{2}{*}{ Eurih } & $\begin{array}{l}\text { Diabetes, } \\
\text { ulcer, body } \\
\text { ache, tonic }\end{array}$ & $\begin{array}{l}\text { Water decoction of } \\
\text { root }\end{array}$ \\
\hline & & & Waist pain & $\begin{array}{l}\text { Squeezed leaves are } \\
\text { rubbed on waist }\end{array}$ \\
\hline & \multirow{5}{*}{$\begin{array}{l}\text { Oryza sativa } \mathrm{L} \text {. var. } \\
\text { formaglotinosa } \\
\text { Oryza sativa } \mathrm{L} \text {. var. } \\
\text { formaglutinosa }\end{array}$} & Ketan bodas & Hemorrhoids & The seed is cooked \\
\hline & & \multirow[t]{4}{*}{ Ketan hideung } & Bleeding & $\begin{array}{l}\text { The seeds are brewed } \\
\text { in hot water, filtered } \\
\text { and used for drinking }\end{array}$ \\
\hline & & & Bruised skin & $\begin{array}{l}\text { Chewed seed is } \\
\text { applied to bruise }\end{array}$ \\
\hline & & & Skincare & $\begin{array}{l}\text { Seed paste is used as } \\
\text { mask }\end{array}$ \\
\hline & & & $\begin{array}{l}\text { Post partum } \\
\text { care }\end{array}$ & $\begin{array}{l}\text { Rice soaked water is } \\
\text { used for drinking }\end{array}$ \\
\hline & Oryza sativa L. var. red & Pare beureum & Diabetes & $\begin{array}{l}\text { Husk powder is mixec } \\
\text { with hot water and } \\
\text { used for drinking }\end{array}$ \\
\hline & $\begin{array}{l}\text { Serycocalyx crispus } \\
\text { (L.)Bremek }\end{array}$ & Pecah beling & $\begin{array}{l}\text { Urinary } \\
\text { retention, } \\
\text { body ache }\end{array}$ & $\begin{array}{l}\text { Water decoction of } \\
\text { leaves }\end{array}$ \\
\hline & \multirow[t]{4}{*}{$\begin{array}{l}\text { Cymbopogon citratus } \\
\text { (DC.)Stapf. }\end{array}$} & \multirow[t]{4}{*}{ Sereh } & $\begin{array}{l}\text { Digestive } \\
\text { health }\end{array}$ & $\begin{array}{l}\text { Pounded young stem } \\
\text { and root are mixed } \\
\text { with sugar }\end{array}$ \\
\hline & & & Rheumatism & $\begin{array}{l}\text { Pounded stem and roc } \\
\text { are smeared on } \\
\text { affected part }\end{array}$ \\
\hline & & & $\begin{array}{l}\text { Post partum } \\
\text { care }\end{array}$ & $\begin{array}{l}\text { Water decoction of } \\
\text { stem }\end{array}$ \\
\hline & & & Vertigo & $\begin{array}{l}\text { Water decoction of } \\
\text { leaves is applied to th } \\
\text { body }\end{array}$ \\
\hline Polygalaceae & Polygala paniculata & Jukut pepeo & Hernia & $\begin{array}{l}\text { All part of plant is } \\
\text { steamed, pounded, } \\
\text { added with salt, taped } \\
\text { on stomach }\end{array}$ \\
\hline Punicaceae & Punica granatum L. & Delima & Hypertension & $\begin{array}{l}\text { Water decoction of } \\
\text { leaves }\end{array}$ \\
\hline
\end{tabular}




\begin{tabular}{|c|c|c|c|c|}
\hline Family & Scientific name & Vernacular name & $\begin{array}{c}\text { Ailment } \\
\text { treated }\end{array}$ & $\begin{array}{l}\text { Plant part used and } \\
\text { processing }\end{array}$ \\
\hline Rosaceae & Rosa $\times$ centifolia $\mathrm{L}$. & Ros & $\begin{array}{l}\text { Female } \\
\text { contraception }\end{array}$ & $\begin{array}{l}\text { Water decoction of } \\
\text { flower }\end{array}$ \\
\hline \multirow[t]{6}{*}{ Rubiaceae } & Morinda citrifolia $\mathrm{L}$. & Cangkudu & $\begin{array}{l}\text { Diabetes, } \\
\text { tonic, ulcer } \\
\text { Vertigo }\end{array}$ & $\begin{array}{l}\text { Fruit is shredded to } g \epsilon \\
\text { the extract } \\
\text { Water decoction of } \\
\text { leaves is applied to } \\
\text { body }\end{array}$ \\
\hline & $\begin{array}{l}\text { Uncaria gambir } \\
\text { (Hunter.) Roxb. }\end{array}$ & Gambir & Toothache & $\begin{array}{l}\text { Squeezed leaves are } \\
\text { rubbed on teeth }\end{array}$ \\
\hline & $\begin{array}{l}\text { Gardenia jasminoides } \\
\text { Ellis. }\end{array}$ & Kaca piring & Fever & $\begin{array}{l}\text { The sap of squeezed } \\
\text { leaves is collected, } \\
\text { filtered and clotted }\end{array}$ \\
\hline & $\begin{array}{l}\text { Plectronia horrida } \\
\text { Scum. }\end{array}$ & Kaliyage & $\begin{array}{l}\text { Post partum } \\
\text { care }\end{array}$ & $\begin{array}{l}\text { Powder of dried leave } \\
\text { is served as herbal } \\
\text { drink }\end{array}$ \\
\hline & Musaenda frondosa L. & Kingkilaban & Eye irritation & $\begin{array}{l}\text { Leaves sap is dropped } \\
\text { into the eyes }\end{array}$ \\
\hline & & & $\begin{array}{l}\text { Post partum } \\
\text { care }\end{array}$ & $\begin{array}{l}\text { Powder of dried leave } \\
\text { is served as herbal } \\
\text { drink }\end{array}$ \\
\hline \multirow[t]{3}{*}{ Rutaceae } & Citrus maxima Merr. & Jeruk bali & Fever & $\begin{array}{l}\text { Water decoction of } \\
\text { fruit }\end{array}$ \\
\hline & $\begin{array}{l}\text { Citrus sinensis (L.) } \\
\text { Osbeck }\end{array}$ & Jeruk manis & Hypertension & Fruit is eaten directly \\
\hline & $\begin{array}{l}\text { Citrus aurantifolia } \\
\text { (Christm.\&Panz.) } \\
\text { Swingle }\end{array}$ & Jeruk nipis & Cough, fever & $\begin{array}{l}\text { The juice is mixed } \\
\text { with soy sauce and } \\
\text { warm water }\end{array}$ \\
\hline Sapindaceae & $\begin{array}{l}\text { Cardiospermum } \\
\text { halicacabum } \mathrm{L} .\end{array}$ & Paria hutan & Skin ulcer & $\begin{array}{l}\text { Squeezed leaves are } \\
\text { smeared on skin }\end{array}$ \\
\hline \multirow[t]{8}{*}{ Solanaceae } & Nicotiana tabacum L. & Bako' & Stomachache & $\begin{array}{l}\text { Leaves are brewed in } \\
\text { hot water, filtered, \& } \\
\text { used for drinking }\end{array}$ \\
\hline & Physalis angulata $\mathrm{L}$. & Cecendet & $\begin{array}{l}\text { Fever, body } \\
\text { ache, } \\
\text { menstruation } \\
\text { cramp, post } \\
\text { partum care, } \\
\text { urinary } \\
\text { retention, tonic }\end{array}$ & $\begin{array}{l}\text { Water decoction of } \\
\text { leaves }\end{array}$ \\
\hline & & & Wound & $\begin{array}{l}\text { Pounded leaves are } \\
\text { applied to the wound }\end{array}$ \\
\hline & Capsicum frutescens $\mathrm{L}$. & Cengek & Cut skin & $\begin{array}{l}\text { Pounded fruit is } \\
\text { applied to the wound }\end{array}$ \\
\hline & & & $\begin{array}{l}\text { Post partum } \\
\text { care, headache }\end{array}$ & $\begin{array}{l}\text { Served as food } \\
\text { seasoning }\end{array}$ \\
\hline & $\begin{array}{l}\text { Solanum tuberosum } \\
\text { (L.) }\end{array}$ & Kentang & $\begin{array}{l}\text { Digestive } \\
\text { health }\end{array}$ & Boiled tuber \\
\hline & Solanum melongena $\mathrm{L}$. & Terong peuheur & $\begin{array}{l}\text { Erectile } \\
\text { dysfunction }\end{array}$ & $\begin{array}{l}\text { Pounded seed is } \\
\text { smeared on penis }\end{array}$ \\
\hline & Cypomandra betacea & Terong walanda & Toothache & Burned in a \\
\hline
\end{tabular}




\begin{tabular}{|c|c|c|c|c|}
\hline Family & Scientific name & Vernacular name & $\begin{array}{c}\text { Ailment } \\
\text { treated }\end{array}$ & $\begin{array}{l}\text { Plant part used and } \\
\text { processing }\end{array}$ \\
\hline & Sendtn. & & & $\begin{array}{l}\text { earthenware vessel, } \\
\text { the steam is rubbed or } \\
\text { teeth }\end{array}$ \\
\hline Sterculiaceae & $\begin{array}{l}\text { Melochia umbellata } \\
\text { O.Stapf. }\end{array}$ & Bintinu & Fracture & $\begin{array}{l}\text { The bark is tied to the } \\
\text { broken part }\end{array}$ \\
\hline Theaseae & Thea sinensis L. & Teh & Diarrhea & $\begin{array}{l}\text { Water decoction of } \\
\text { leaves }\end{array}$ \\
\hline Thymelaeceae & $\begin{array}{l}\text { Phaleria Macrocarpa } \\
\text { (Scheff.)Boerl. }\end{array}$ & Mahkota dewa & Tonic & $\begin{array}{l}\text { Sliced fruit is dried, } \\
\text { served as tea }\end{array}$ \\
\hline \multirow[t]{2}{*}{ Urticaceae } & $\begin{array}{l}\text { Villebrunea rubescens } \\
\text { (BI.) BI. }\end{array}$ & Nangsi & $\begin{array}{l}\text { Digestive } \\
\text { health }\end{array}$ & $\begin{array}{l}\text { Leaves are eaten } \\
\text { directly }\end{array}$ \\
\hline & $\begin{array}{l}\text { Pilea melastomoides } \\
\text { (Poir.) BI. }\end{array}$ & Poh pohan & rheumatism & $\begin{array}{l}\text { Water decoction of } \\
\text { leaves }\end{array}$ \\
\hline \multirow[t]{2}{*}{ Verbenaceae } & $\begin{array}{l}\text { Stachytarpheta indica } \\
\text { (L.) Vahl. }\end{array}$ & Jarong & $\begin{array}{l}\text { Post partum } \\
\text { care }\end{array}$ & $\begin{array}{l}\text { Powder of dried root } \mathrm{i} \\
\text { served as herbal drink }\end{array}$ \\
\hline & & & Swollen body & $\begin{array}{l}\text { Water decoction of all } \\
\text { part of plant }\end{array}$ \\
\hline \multirow[t]{12}{*}{ Zingiberaceae } & Kaempferia galanga L. & Cikur & $\begin{array}{l}\text { Bump, boil, } \\
\text { luxation, } \\
\text { bruise, } \\
\text { headache, } \\
\text { stomachache }\end{array}$ & $\begin{array}{l}\text { Pounded rhizome is } \\
\text { rubbed on affected } \\
\text { part }\end{array}$ \\
\hline & & & Fever & $\begin{array}{l}\text { Water decoction of } \\
\text { rhizome }\end{array}$ \\
\hline & & & $\begin{array}{l}\text { Digestive } \\
\text { health }\end{array}$ & $\begin{array}{l}\text { Pounded rhizome is } \\
\text { mixed with sugar }\end{array}$ \\
\hline & & & $\begin{array}{l}\text { Post partum } \\
\text { care }\end{array}$ & $\begin{array}{l}\text { Powder of dried } \\
\text { rhizome is served as } \\
\text { herbal drink }\end{array}$ \\
\hline & & & Tonic & $\begin{array}{l}\text { Water decoction of } \\
\text { rhizome }\end{array}$ \\
\hline & $\begin{array}{l}\text { Nicolaia speciosa (BI.) } \\
\text { Horan }\end{array}$ & Honje & Headache & $\begin{array}{l}\text { Water decoction of } \\
\text { stem }\end{array}$ \\
\hline & $\begin{array}{l}\text { Zingiber officinale } \\
\text { Roxb. }\end{array}$ & Jahe & $\begin{array}{l}\text { Post partum } \\
\text { care }\end{array}$ & $\begin{array}{l}\text { Pounded rhizome is } \\
\text { mixed with warm } \\
\text { charcoal wrapped witl } \\
\text { banana leaves to sit or }\end{array}$ \\
\hline & & & Rheumatism & $\begin{array}{l}\text { Pounded rhizome is } \\
\text { smeared on affected } \\
\text { part }\end{array}$ \\
\hline & & & Common cold & $\begin{array}{l}\text { Shredded rhizome is } \\
\text { mixed with coconut } \\
\text { oil, smeared on the } \\
\text { body }\end{array}$ \\
\hline & & & Cough & $\begin{array}{l}\text { Extract of shredded } \\
\text { rhizome is used for } \\
\text { drinking }\end{array}$ \\
\hline & $\begin{array}{l}\text { Amomum cardamomum } \\
\text { Willd. }\end{array}$ & Kapulaga & Asphyxiate & $\begin{array}{l}\text { Water decoction of } \\
\text { fruit }\end{array}$ \\
\hline & Curcuma xanthorriza & Koneng ageung & Asthma, & Water decoction of \\
\hline
\end{tabular}




\begin{tabular}{|c|c|c|c|c|}
\hline Family & Scientific name & Vernacular name & $\begin{array}{c}\text { Ailment } \\
\text { treated }\end{array}$ & $\begin{array}{l}\text { Plant part used and } \\
\text { processing }\end{array}$ \\
\hline \multicolumn{2}{|r|}{ Roxb. } & & $\begin{array}{l}\text { diarrhea, body } \\
\text { ache, ulcer, } \\
\text { post partum } \\
\text { care, tonic } \\
\text { Ulcer, appetite } \\
\text { booster }\end{array}$ & $\begin{array}{l}\text { The powder of dried } \\
\text { rhizome is served as } \\
\text { drink }\end{array}$ \\
\hline & \multirow[t]{2}{*}{$\begin{array}{l}\text { Curcuma zedoaria } \\
\text { (Berg.) Roscoe }\end{array}$} & \multirow[t]{2}{*}{ Koneng bodas } & Tonic & $\begin{array}{l}\text { Water decoction of } \\
\text { rhizome }\end{array}$ \\
\hline & & & $\begin{array}{l}\text { Post partum } \\
\text { care }\end{array}$ & $\begin{array}{l}\text { Powder of dried } \\
\text { rhizome is served as } \\
\text { herbal drink }\end{array}$ \\
\hline & $\begin{array}{l}\text { Curcuma aeruginosa } \\
\text { Roxb. }\end{array}$ & Koneng hideung & $\begin{array}{l}\text { Diarrhea, } \\
\text { ulcer, } \\
\text { dizziness, } \\
\text { body ache, } \\
\text { tonic }\end{array}$ & $\begin{array}{l}\text { Water decoction of } \\
\text { rhizome }\end{array}$ \\
\hline & \multirow[t]{4}{*}{$\begin{array}{l}\text { Curcuma domestica } \\
\text { Vahl. }\end{array}$} & \multirow[t]{4}{*}{ Koneng sayur } & $\begin{array}{l}\text { Diarrhea, } \\
\text { appetite } \\
\text { booster, ulcer }\end{array}$ & $\begin{array}{l}\text { Extract of shredded } \\
\text { rhizome is used for } \\
\text { drinking }\end{array}$ \\
\hline & & & Eczema & $\begin{array}{l}\text { Pounded rhizome is } \\
\text { smeared on skin }\end{array}$ \\
\hline & & & $\begin{array}{l}\text { Digestive } \\
\text { health } \\
\text { stomachache }\end{array}$ & $\begin{array}{l}\text { Pounded rhizome is } \\
\text { mixed with sugar } \\
\text { Water decoction of } \\
\text { leaves }\end{array}$ \\
\hline & & & $\begin{array}{l}\text { Post partum } \\
\text { care, tonic }\end{array}$ & $\begin{array}{l}\text { Water decoction of } \\
\text { rhizome }\end{array}$ \\
\hline & \multirow[t]{2}{*}{$\begin{array}{l}\text { Languas galanga (L.) } \\
\text { Stuntz. }\end{array}$} & \multirow[t]{2}{*}{ Laja } & Skin ulcer & $\begin{array}{l}\text { Pounded rhizome is } \\
\text { smeared on skin }\end{array}$ \\
\hline & & & Swollen feet & $\begin{array}{l}\text { Shredded rhizome is } \\
\text { added to warm cinder } \\
\text { and smeared }\end{array}$ \\
\hline & $\begin{array}{l}\text { Zingiber zerumbet (L.) } \\
\text { J.E.Smith }\end{array}$ & Lampuyang & $\begin{array}{l}\text { Post partum } \\
\text { care }\end{array}$ & $\begin{array}{l}\text { Rhizome is dried, } \\
\text { crushed, added with } \\
\text { water and used for } \\
\text { drinking; fresh } \\
\text { rhizome is steamed, } \\
\text { smeared on stomach }\end{array}$ \\
\hline & Impatiens balsamina $\mathrm{L}$. & Pacar putih & $\begin{array}{l}\text { Female } \\
\text { contraception }\end{array}$ & $\begin{array}{l}\text { Water decoction of } \\
\text { flower }\end{array}$ \\
\hline & \multirow{4}{*}{$\begin{array}{l}\text { Costus speciosus } \\
\text { (Kuen.) J.E.Smith } \\
\text { Zingiber purpureum } \\
\text { Roxb. }\end{array}$} & Pacing & Hypertension & $\begin{array}{l}\text { Extract of stem is user } \\
\text { for drinking }\end{array}$ \\
\hline & & \multirow[t]{3}{*}{ Panglay } & $\begin{array}{l}\text { Asthma, } \\
\text { asphyxiate, flu } \\
\text { in children }\end{array}$ & $\begin{array}{l}\text { Pounded rhizome is } \\
\text { smeared on chest }\end{array}$ \\
\hline & & & $\begin{array}{l}\text { Baby care } \\
\text { after birth }\end{array}$ & $\begin{array}{l}\text { Squeezed leaves are } \\
\text { smeared on body }\end{array}$ \\
\hline & & & $\begin{array}{l}\text { Post partum } \\
\text { care }\end{array}$ & $\begin{array}{l}\text { Pounded rhizome is } \\
\text { smeared on body }\end{array}$ \\
\hline
\end{tabular}




\begin{tabular}{|c|c|c|c|c|}
\hline \multirow[t]{4}{*}{ Family } & Scientific name & Vernacular name & $\begin{array}{c}\text { Ailment } \\
\text { treated }\end{array}$ & $\begin{array}{l}\text { Plant part used and } \\
\text { processing }\end{array}$ \\
\hline & $\begin{array}{l}\text { Boesenbergia } \\
\text { pandurata (Roxb.) }\end{array}$ & Temu kunci & Fever in baby & $\begin{array}{l}\text { Pounded leaves are } \\
\text { smeared on body }\end{array}$ \\
\hline & Schlecht. & & $\begin{array}{l}\text { Digestive } \\
\text { health }\end{array}$ & $\begin{array}{l}\text { Cleaned rhizome is } \\
\text { eaten raw }\end{array}$ \\
\hline & & & $\begin{array}{l}\text { Ulcer, } \\
\text { hypertension }\end{array}$ & $\begin{array}{l}\text { Extract from shredded } \\
\text { rhizome }\end{array}$ \\
\hline
\end{tabular}

Illnesses suffered commonly by the people of Kampung Dukuh were cough and fever. Nevertheless, they also recognized medicinal plants to cure other complicated illnesses such as diabetes, hypertension, cardiac complication, asthmatic and intoxication. Figure 2 shows the number of species used in treating disease usually suffered by people of Kampung Dukuh. The highest total number of plant used was on pre and postpartum care. The traditional method applied by people of Kampung Dukuh on pre and postpartum care is specifically discussed in the next subchapter.

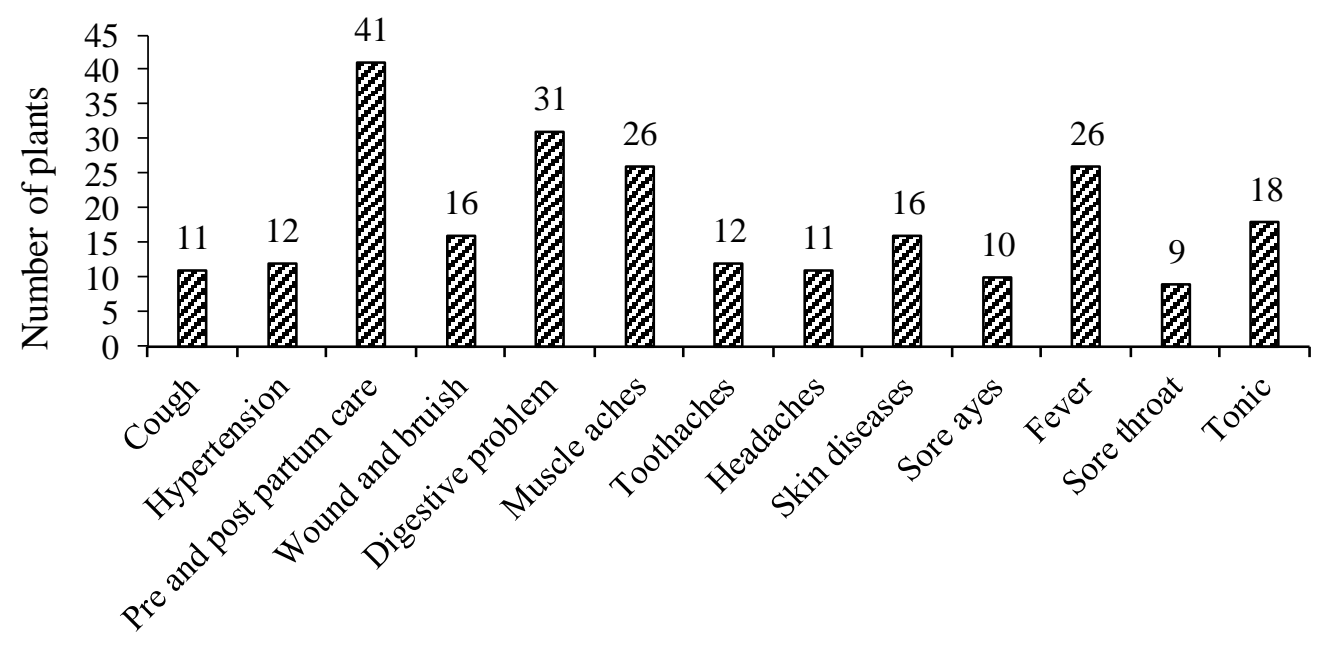

Type of illnesses

Figure 2. Number of species used as medicines of each group of health conditions

Plant Part Used and Mode of Preparation

Plant parts used by people of Kampung Dukuh as medicines were root, stem, seed, fruit, flower, leaf, rhizome, and tuber (Figure 3). Leaf was part of plant mostly used by the community. Most of the plant-based medicines known only consisted of one part of a plant species, for instance, Ageratum conyzoides L. (leaf), Physalis angulata L. (leaf), Kaempferia galanga L. (rhizome), Oryza sativa L. (var.) formaglutinosa (seed) and Alstonia scholaris (L.) R.Br. (stem). It was documented that the community also used more than one plant part of some species. Root and stem of Imperata cylindrica were used in curing diabetes, dyspepsia, muscle aches, also as tonic. Almost every part of Carica papaya L. was recognized as medicines, starting from the roots, leaves, sap of leaves, stems, fruit, flower and even seeds to treat ailments such as hypotension, malaria, postpartum care, body aches, toothaches, digestive complications, and headaches. Leaf extract was used for drinking by processed it into breastmilk supply booster and tonic. The stem, root, and leaf of Cymbopogon citratus (DC.) Stapf were used to treat digestive complication, rheumatic disease, pre and postpartum care and vertigo. 


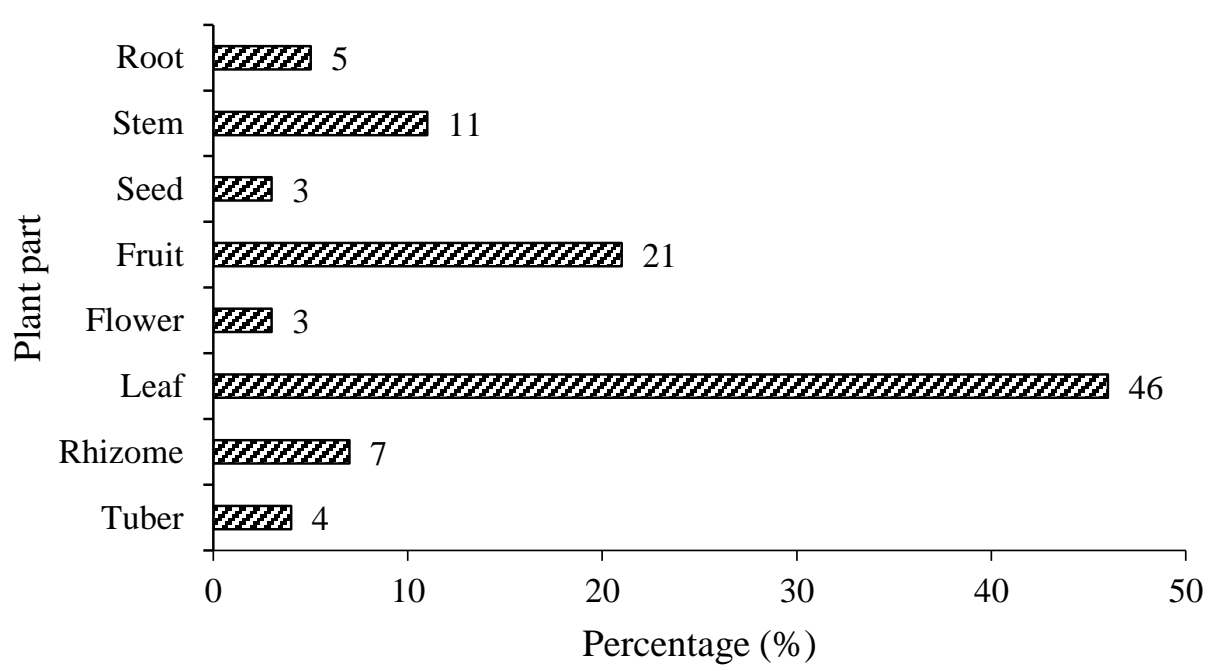

Figure 3. Percentages of plant part used

The community classified two types of treatment; namely external and internal treatment. Ailments treated externally were like skin pain, toothache, earache, and sore eyes. Internal treatment was the type of treatment applied by eating or drinking single or mixture of the medicinal herbs orally. The medicine could be in the forms of extract, paste or raw herbs. Most of the external ailments were treated with a single plant composition only. Most part used in curing wound and skin disease was leaf and taken from one type of plant. For example, Dysoxylum decandrum Merr. was used as medicine for the diabetic wound. Some people in Kampung Dukuh were even found to cultivate this species in their garden and forest edge. The preparation of this treatment was performed by crushing the ingredients. Firstly, the leaves were washed and then crushed into small pieces. Crushed paste smeared directly onto the wound thickly. The wound was covered and tied up with a bandage. The coat was changed every day until the wound dried. Resin and sap of plant were also used for external treatment, particularly for ears and eye ailment.

Modes of preparation of medicinal plants for internal ailment known were generally done by boiling the plant to get the extract or by scrapping it to get the concentrate. For internal ailments, the composition of medicines mostly consisted of more than one plant. For instance, to make a traditional tonic, they boiled various parts of plant such as rhizome of
Curcuma xanthorriza Roxb., Curcuma zedoaria (Berg.) Roscoe, Curcuma aeruginosa Roxb. and Curcuma domestica Vahl; the leaf of Physalis angulata L., Blumea balsamifera (L.) D.C., Desmodium triquetrum D.C., Orthosiphon aristatus (BI.) Miq., Moringa oleifera Lamk., Eupatorium odoratum; the root of Carica papaya L., Imperata cylindrica, Areca catechu L.; tree bark of Allamanda cathartica L. and stem of Tinospora tubreculata Beumee.

\section{Traditional Methods for Pre and Postpartum Care}

In Kampung Dukuh, pregnant women would visit the paraji firstly on the fourth month of pregnancy. On this visit, paraji would check the pregnancy condition and suggest the expectant to drink such kind of tonic consists of Curcuma domestica Vahl. mixed with honey and egg. On seventh month of pregnancy, the expectant would visit paraji gradually every two weeks. In this phase, paraji would start to give the expectant a massage to make sure the baby is in the proper position inside the uterus. On the final stage of pregnancy, before delivery, the patient would be suggested to drink the boiled water of Ceiba petandra (L.) Gaertn that was considered effective in enhancing contraction during labor. Paraji smeared the klentik oil (traditional virgin oil) mixed with the crushed of Alium cepa $\mathrm{L}$. and Zingiber purpureum Roxb. on the belly of the expectant. This concoction will 
result in colic effect to patients that aims to accelerate the birth process.

Paraji was also responsible for newborn care. Paraji would smear the newborn with the concentrate of Zingiber purpureum Roxb. especially on the heel, ankle, and stomach to warm the baby. The post-partum mother would be given the concoction known as opat puluh rupi which consists of 40 species of plants. These plants were washed and dried then crushed into powder. Later, warm water was poured into the powder, and the mix was given to the patient as herbal drink at dose of one tablespoon per 8 hours. Some ingredients of this concoction were rhizomes of Kaempferia galanga L, Zingiber officinale Roxb., Curcuma zedoaria (Berg.) Roscoe, Boesenbergia pandurata (Roxb.) Schlecht., and Zingiber zerumbet (L.)J.E.Smith; roots of Cocos nucifera L, Areca catechu L., Physalis angulata L., Stachytarpheta indica (L.) Vahl., Imperata cylindrica (Ness) C.E.Hubb. and Arenga pinnata (Wurmb.) Merr; tree barks of Alstonia scholaris (L.) $\mathrm{R} . \mathrm{Br}$ and Allamanda cathartica L.; and leaves of Blumea balsamifera (L.) D.C., Dolichandrone spathecea (L.f.)K. Schum., Musaenda frondosa L., Erythrina subumbrans (Hassk.) Merr. and Cymbopogon citratus (DC.) Stapf.

From the interview, it was found that labor complication of severe bleeding was mostly occurred. Therefore, paraji would give the patient extract of Piper betle and Euphorbia hirta L. mixed with soaked water of black rice (Oryza sativa L. var formaglutinosa). To avoid infection and to tighten up the vagina, the mother would sit on a hot pad made of ash wrapped with the leaf of Ricinus communis L. This treatment was done every day until day $40^{\text {th }}$ after delivery. Moreover, to remove the stretch mark on belly skin after giving birth, mothers would smear their skin with crushed rhizome of Zingiber zerumbet (L.) J.E.Smith and leaf of Eleusine indica Gaertn. These two ingredients were pounded with lime. For contraception, the women of Kampung Dukuh used to drink the water decoction of banana blossom, rose flower, and flower of Impatiens balsamina L.

\footnotetext{
Distribution of Medicinal Plant in The Study Area
}

People of Kampung Dukuh obtained medicinal plants from five locations; buruan (37 species), huma (6 species), kebon (72 species), leuweung (42 species), and sidewalk (25 species). Buruan or home garden is the land around the house devoted to planting any kinds of plants including medicinal ones. Leuweung is the forest. The plants obtained from the forest were commonly of wooden materials (trees and shrubs), such as Alstonia scholaris (L.) R.Br., Allamanda cathartica L., Melochia umbellata O.Stapf., and Cardiospermum halicacabum L. Kebon is a semi cultivated land located on the forest edge, a hilly region far from hamlet. Kebon in Kampung Dukuh was an agroforestry typical. Plants were variedly grown in kebon from fruit to timber trees. Wild medicinal plants such Ageratum conyzoides L., Mikania scandens Willd, and Euphorbia hirta L. were also found in kebon. Huma is the cultivated land specialized in planting staple food plants such as rice, maize, and sweet potatoes. Huma is distinguished from kebon by looking at the composition of the plant. Huma is dominated by one kind of main plant. The wooden plant is rarely found in huma. Based on interviews and observation, $59 \%$ of medicinal plants in Kampung Dukuh were wild plants and the remaining $41 \%$ were cultivated plants. Most wild medicinal plants were obtained from kebon, leuwueng, and sidewalk. Medicinal plants were intentionally planted in buruan and kebon.

\section{DISCUSSION}

This study has revealed important information on medicinal plants used by traditional people, traditional healers, midwife, and elder herbalists of Kampung Dukuh, West Java, to treat various ailments. It also demonstrated the vital role that medicinal plants play an essential part in the health care of these people. For local community like people of Kampung Dukuh, forest is the biggest part of their life. The community would utilize forest resources to fulfill their daily needs. Besides forests, other landscapes like farm fields, settlements, and even shrub filled fields are also utilized to support their life. Traditional communities who constantly interact with the surrounding environment tend 
to have deep local knowledge related to the surrounding resources (Gadgil, Berkes, \& Folke, 1993; Balick \& Cox, 1996). In daily life, traditional people are strongly related to their natural environment, especially plants. Plants are used as food, clothing, shelter construction, medicine and so on. In Kampung Dukuh, some plants were also considered sacred or magical and used in traditional ceremonies or rituals and social activities of local communities.

Traditional people of Kampung Dukuh community were found to have knowledges about the use of plants for traditional medicine since they recognized 131 species of medicinal plants. We also calculated the number of species known by traditional people of other traditional ethnics in Indonesia, namely those from two nearby traditional hamlets of Sundanese ethnic in Kampung Kuta, Ciamis (Dwiartama, 2005) and in Kampung Naga, Tasikmalaya (Nurmalasari, Sukarsa, \& Hidayah, 2012) which resulted in 148 and 108 species, respectively. Most dominant family used in Kampung Dukuh was Zingiberaceae with rhizome as the plant part mostly used. Most species on Zingiberaceae in Kampung Dukuh were cultivated.

Every region has its specific plant utilization systems. The system is related to the plant diversity in each region. Traditional people tend to practice a sustainable management system in utilizing the natural ecosystem to support long term humankind needs (Swanson, 1995). Traditional management in using natural resources can clarify the relationship between the conservation system and the using of natural resources. The people of Kampung Dukuh were observed to have particular characteristics in utilizing plants as medicines. These characteristics can be described into three aspects: location the plant obtained, plant status (wild or cultivated), and plant part used.

People of Kampung Dukuh obtained medicinal plants mostly from their agroforestry field (kebon) dominated with fruit/food trees such as jackfruit (Artocarpus heterophyllus), durian (Durio zibethinus), rambutan (Nephelium lappaceum), coffee (Coffea sp.), and pepper (Piper nigrum). Wild plant considered as medicine was allowed to grow in kebon, thus the majority of medicinal plants could be found in this location. The concept of kebon according to people of Kampung Dukuh related to the location to obtain medicinal plants was slightly different from other community in comparable research. For example, Menyah Tribe in Arfak Mountain knew kebon as mekeni. Mekeni is land planted with fruit trees dominated with cocoa. However, Menyah Tribe got the majority of the medicinal plants from primary forest, called Merenda (Moeljono, 1998). Other community in Dheeraa, Ethiopia, obtained $92 \%$ of their medicinal plants from the natural vegetation ecosystem, indicating that the local community of Dheeraa did not practice the medicinal plant planting in cultivation area like home garden or farm (Wondimu, Asfaw, \& Kelbessa, 2007).

More than $40 \%$ of medicinal plants were planted by the community. Most of domesticated medicinal plants were planted in home garden. In Kampung Dukuh, home garden was where many kinds of wild plants, semi-cultivated plants, and cultivated plants grow together. Home garden has a vital role in many aspects including the economy, ecology, social and culture (High \& Shackleton, 2000; Méndez, Lok, \& Somarriba, 2001; Senanayake, Sangakkara, Pushpakumara, \& Stamp, 2009). Each home garden has a unique structure, function, and composition depending on the natural ecological states (Galhena, Freed, \& Maredia, 2013). For instance, Batak Karo sub-ethnic in North Sumatra, Indonesia, intentionally planted a total of 85 species of plants as traditional medicine and foodstuffs in their home garden with most plant part used was fruit (Silalahi \& Nisyawati, 2018). Anak Rawa Ethnic in Riau planted 8 species of of home garden typical plant aimed as medical purpose, such as Brucea javanica, Globba pendula, Flemingia strobilifera, Trema tomentosa, Ilex cymosa, Timonius sp., Uncaria sp., and Dillenia excelsa (Utami, Zuhud, \& Hikmat, 2019).

The people of Kampung Dukuh also obtained various kinds of medicinal plants from the forest, yet it was not the most proportion. They were allowed to take the natural substance in the forest under the control of kuncen (local forest caretaker) rules. 
This control management of natural resources by kuncen aimed to keep up the stability of plant diversity of the local forest. The concept of sacred forest (hutan larangan) shows the system of resource patterning of the community to ensure their natural sustainability. Conversely, the majority of traditional healers of many places and community in the world do not believe that the availability of medicinal plants in nature is going to drop (Swanson, 1995). They believe that the medicinal plant is the gift from the supreme being and will always be available in nature. This concept limits the effort of the community to maintain the number of medicinal plant species in their natural state.

The conservation effort in maintaining the sustainability of medicinal plants in nature is closely related to the plant part used as medicine. Plant parts that should be restricted in use are root, stem, tree bark, and tuber. The medicinal plant part mostly used by the people of Kampung Dukuh was leaves. The use of leaves as medicines has a limited impact on plant for their potency and fast regeneration. Medicinal plants were either used singly or as a mixture of more than one species to make a specific efficacy. The use of various plants for the effective treatment of one particular disease could be due to the synergistic effect. One plant could be used for many diseases probably due to the presence of various metabolites and also the fact that the same molecule can be active on different pathogens (Namukobe et al., 2011). A comparable study in Kenya also showed that leaves were preferred to be used as medicine to other parts of the plant (Jeruto, Lukhoba, Ouma, Otieno, \& Mutai, 2008). As comparison, Anak Rawa community in Riau mostly used root part. This community believed that the root has the strongest ingredient and last longer on the body than other plant parts (Utami et al., 2019). Other nearby community, i.e. Batak Karo sub ethnic utilized fruit part the most (Silalahi \& Nisyawati, 2018). Leaf part that was mostly used by Kampung Dukuh community indicated that that community respected the sustainalibily of biological resource and valuable knowledge in recognizing plants as medicines. Overall, they can integrate the culture of the utilization of plants as traditional medicine with the effort to conserve plant diversity in nature.

\section{CONCLUSION}

Based on this study, it is concluded that local knowledge of Dukuh people on medicinal plant diversity was still preserved. People of Kampung Dukuh recognized 131 species of medicinal plants from 51 families. Moreover, families with the most number of species were Zingiberaceae (14 species), Poaceae (8 species), Asteraceae (7 species), Fabaceae (6 species), and Solanaceae (6 species). The community classified two types of treatment; external and internal treatment. For the external treatment, the processing was mostly by crushing from a single plant composition only, while for the internal treatment, the processing was generally by boiling the plant to get the extract or by scrapping it to get the concentrate, mostly from two or more mixture of plants. The most number of plants used were observed on pre and postpartum care. Based on the plant part used and the distribution of medicinal plant found, it is reflected that their system in using plants as traditional medicine was specific. The people of kampung Dukuh can integrate the culture of plant using as medicine with the effort to conserve their plant diversity.

\section{REFERENCES}

Backer, C. A., \& van den Brink, R. C. B. (1965). Flora of Java (Spermatophytes only) vol. i-iii. Groningen: WoltersNoordhoff N.V.

Balick, M. J., \& Cox, P. A. R. (1996). Plants, people and culture: The science of ethnobotany. New York: Scientific American Library.

Balick, M. J. (2007). Traditional knowledge: Lessons from the past, lessons for the future. In: C. R. McManis (Eds.), Biodiversity \& the law: Intellectual property, biotechnology \& traditional knowledge (pp. 280-296). London: Earthscan.

Bodeker, G. (2000). Indigenous medical knowledge: The law and politics of protection. Oxford: Oxford Intellectual Property Research Centre. 
Brosi, B. J., Balick, M. J., Wolkou, R., Lee, R., \& Kostka, M. (2007). Cultural erosion and biodiversity: Canoe-making knowledge in Pohnpei, Micronesia. Conservation Biology, 21, 875-879. doi: 10.1111/j.1523-1739.2007.00654.x

Dwiartama, A. (2005). Analisis pengetahuan tradisional masyarakat adat Kampung Kuta Kabupaten Ciamis mengenai pemanfaatan tumbuhan untuk pengobatan (Skripsi sarjana). Sekolah Ilmu dan Teknologi Hayati (SITH) Institut Teknologi Bandung (ITB), Bandung, Indonesia.

Gadgil, M., Berkes, F., \& Folke, C. (1993). Indigenous knowledge for biodiversity conservation. Ambio, 22(2-3), 151-156.

Galhena, D. H., Freed, R., \& Maredia, K. M. (2013). Home gardens: A promising approach to enhance household food security and wellbeing. Agricultral \& Food Security, 2(8), 1-13.

Gunawan, R., Ramadhan, U. G., Iskandar, J., \& Partasamita, R. (2018). Local knowledge of utilization and management of sugar palm (Arenga pinnata) among Cipanggulaan People of Karyamukti, Cianjur (West Java, Indonesia). Biodiversitas, 19(1), 93-105.

Hernani. (2011). Perkembangan biofarmaka sebagai obat herbal untuk kesehatan. Buletin Teknologi Pascapanen Pertanian 7(1), 20-29.

High, C., \& Shackleton, C. M. (2000). The comparative value of wild and domestic plant in homegarden of a South African rural village. Agroforestry system, 48, 141-156.

Jeruto, P., Lukhoba, C., Ouma, G., Otieno, D., \& Mutai, C. (2008). An ethnobotanical study of medicinal plants used by the Nandi people in Kenya. Journal of Ethnopharmacology, 116, 370-376.

Kaido, T. L., Veale, D. J. H., Havlik, I., \& Rama, D. B. K. (1997). Preliminary screening of plants used in South Africa as traditional herbal remedies during pregnancy and labour. Journal of Ethnopharmacology, 55, 185-191.

Kusumah, S. D. (2017). The traditional medicine of Bugis-Makassar people. Patanjala, 9(2), 245-260.
Martin, G. J. (1995). Ethnobotany: A methods manual. London: Chapman \& Hall.

Méndez, V. E., Lok, R., \& Somarriba, E. (2001). Interdisciplinary analysis of homegardens in Nicaragua: Microzonation, plant use and socioeconomic importance. Agroforestry System, 51, 8596.

Moeljono, S. (1998, May 5-6). Suatu telaah tentang pemanfaatan keanekaragaman jenis tumbuhan oleh masyarakat Suku Menyah di daerah Pegunungan Arfak Kabupaten Manokwari. Paper presented at Prosiding Seminar Nasional Etnobotani III LIPI, Denpasar, Bali, Indonesia.

Munadi, E. (2017). Tanaman obat, sebuah tinjauan singkat. In Z. Salim, \& E. Munadi (Eds.), Info komoditi tanaman obat (pp. 1-7). Jakarta, Indonesia: Badan Pengkajian dan Pengembangan Perdagangan Kementerian Perdagangan Republik Indonesia.

Namukobe, J., Kasenene, J. M., Kiremire, B. T., Byamukama, R., KamatenesiMugisha, M., Krief, S., \& Kabasa, J. D. (2011). Traditional plants used for medicinal purposes by local communities around the Northern sector of Kibale National Park, Uganda. Journal of Ethnopharmacology, 136, 236-245.

Nielsen, I. C. (1992). Flora Malesiana series iSpermatophyta vol. ii: Mimosaceae. Leiden: Rijksherbarium/Hortus Botanicus. Leiden University.

Nurmalasari, N., Sukarsa., \& Hidayah, H. A. (2012). Studi kasus pemanfaatan tumbuhan sebagai obat-obatan tradisional oleh masyarakat adat Kampung Naga di Kabupaten Tasikmalaya (Case study on the use of plants as traditional medicines by the traditional community of Kampung Naga in Tasikmalaya Regency). Biosfera, 29(3), 141-150.

Ochse, J. J., \& van den Brink, R. C. B. (1931). Vegetables of the Dutch East Indies. Java: Archipel Drukkerij Buitenzorg.

Ogata, Y., Kazahara, Y., Mulyadi., Rahmad, A., Jamaludin., Royadi, B., ... Fauzi, A. (1995). Medicinal Herb Index in 
Indonesia $2^{\text {nd }}$ ed. Jakarta: PT. Eisai Indonesia.

Prawira, S. A. (1976). Laporan no. 219. daftar pohon Jawa-Madura (i) Jawa Barat (Report No.219: List of trees of JavaMadura (i) West Java). Bogor: Bagian Botani Hutan, Lembaga Penelitian Hutan.

Rahman, M. H., Fardusi, M. J., \& Reza, M. S. (2011). Traditional knowledge and use of medicinal plants by the Patra tribe community in the North-Eastern Region of Bangladesh. Proceedings of the Pakistan Academy of Sciences, 48(3), 159-167.

Ramirez, C. R. (2007). Ethnobotany and the loss of traditional knowledge in the $21 \mathrm{st}$ century. Ethnobotany Research and Application, 5, 245-247.

Sari, L. O. R. K. (2006). Pemanfaatan obat tradisional dengan pertimbangan manfaat dan keamanan (Utilization of traditional medicine with consideration of the benefits and safety). Majalah Ilmu Kefarmasian, 3(1), 1-7.

Senanayake, R. L., Sangakkara, U. R., Pushpakumara, D. K. N. G., \& Stamp, P. (2009). Vegetation composition and ecological benefits of home gardens in the Meegahakiula Region of Sri Lanka. Tropical Agricultural Research, 21(1), 19.
Silalahi, M., \& Nisyawati. (2018). The ethnobotanical study of edible and medicinal plants in the home garden of Batak Karo sub-ethnic in North Sumatra, Indonesia. Biodiversitas, 19(1), 229-238.

Suatama, I. B. (2019). Multikulturisme usada Bali (Multiculturalism of Bali Usada). Widya Kesehatan, 1(1), 11-17.

Swanson, T. M. (1995). Intellectual property rights and biodiversity conservation 'an interdisciplinary analysis of the values of medicinal plants. Cambridge: Cambridge University Press.

Utami, R. D., Zuhud, E. A. M., \& Hikmat, A. (2019). Etnobotani dan potensi tumbuhan obat masyarakat etnik Anak Rawa Kampung Penyengat Sungai Apit Siak Riau (Medicinal ethnobotany and potential of medicine plants of Anak Rawa Ethnic at The Penyengat Village Sungai Apit Siak Riau). Media Konservasi, 24(1), 40-51.

Weckmüller, H., Barriocanal, C., Maneja, R., \& Boada, M. (2019). Factors affecting traditional medicinal plant knowledge of the Waorani, Ecuador. Sustainability, 11, 1-12.

Wondimu, T., Asfaw, Z., \& Kelbessa, E. (2007). Ethnobotanical study of medicinal plants around 'Dheeraa' town, Arsi Zone, Ethiopia. Journal of Ethnopharmacology, 112, 152-161. 